Chapter 5

\title{
Brain Mapping of Language Processing Using Functional MRI Connectivity and Diffusion Tensor Imaging
}

\author{
Todd L. Richards and Virginia W. Berninger \\ Additional information is available at the end of the chapter \\ http://dx.doi.org/10.5772/56501
}

\section{Introduction}

Because the brain's language systems have no end organs for interacting directly with the external world, language systems work with sensory (ears or eyes) and motor (mouth and hands) systems, which are the only brain systems with direct links to external environment. Liberman contributed to understanding of how the language by ear (listening) and language by mouth (reading) systems work together at the behavioral level and also become integrated to support acquisition of language by eye (reading) [1]. Berninger and colleagues extended the work of Liberman and colleagues at the Haskins Laboratory to language by hand (writing), which is not just a motor skill as many assume [2]. This University of Washington research team also showed that Language by Ear, Language by Mouth, Language by Eye, and Language by Hand are separate, but interacting functional language systems, which draw on common as well as unique processes at the behavioral [3] and brain levels of analysis [4]. Moreover, each of the functional language systems has different levels of organization, ranging from subword, to word, to syntax, to text, and has connections with other brain systems such as working memory, attention and executive functions, and cognitive.

The emerging work on the complex functional language systems that connect with other brain systems illustrates the need for brain imaging methods that not only assess localized brain areas or functions but also their structural and functional connections. First, we discuss how the modern imaging techniques have confirmed knowledge of localized structures and functions first acquired in autopsy studies with patients. Second, we discuss how advances in imaging techniques are adding knowledge about the structural and functional connections among specific functional language systems. 


\subsection{Localized structures and functions}

In early work in neurolinguistics researchers studied people with brain lesions and discovered relationships between the patient's specific language deficit and the location of the lesion. In this way, they discovered that two areas in the brain are involved in language processing: Wernicke's area located in the posterior section of the superior temporal gyrus in the dominant cerebral hemisphere. People with a lesion in this area of the brain develop receptive aphasia, a condition in which there is a major language comprehension impairment, but the capability for speech production remains intact. The other area is Broca's area located in the posterior inferior frontal gyrus of the dominant hemisphere. Patients with a lesion to this area develop expressive aphasia and are unable to produce speech even though they are able to understand other's that they hear [4].

Neurolinguist researchers have adopted non-invasive brain imaging techniques such as functional magnetic resonance imaging and electrophysiology to study language processing in individuals without impairments [5]. For example, in the study of phonological processing, the receptive processing of phonemes in heard words has been localized to Wernicke's area (posterior Brodmann's Area [BA] 22) and BA 40 [6] [7-11], and expressive production of phonemes during speech has been localized to the posterior Broca's area (BAs 44 and 6) [11-15]. Thus, research using these newly developed brain imaging techniques has confirmed what was was classically thought based on patient studies for right-handed individuals: The two major language areas are Broca's area for production of language by mouth [16] and Wernicke's area for comprehension of language by ear [17], which receives input from the ear through the auditory cortex. The arcuate fasciculus, a fiber pathway that originates in the temporal lobe and curves in an anterior/posterior direction to project to the frontal lobe [18], was thought to connect these 2 areas.

Figure 1 that follows shows these important language processing areas of the brain superimposed on a side/surface view of the brain based on more recent non-invasive brain imaging methods. These areas may also play a role in production of language by hand (writing) and comprehension of language by eye (reading), via related processing in angular gyrus and supramarginal gyrus [4].

\section{Brain's structural and functional connectivity}

In 2010 the US National Institute of Health (NIH) announced the Human Connectome Project:

"Knowledge of human brain connectivity will transform human neuroscience by providing not only a qualitatively novel class of data, but also by providing the basic framework necessary to synthesize diverse data and, ultimately, elucidate how our brains work in health, illness, youth, and old age." Included in this connectome is the study of language-related neural connections which enable the brain to perform written and oral language.

Mullen [19] has on online manual that defines several important terms used in research about structural and functional networks. 


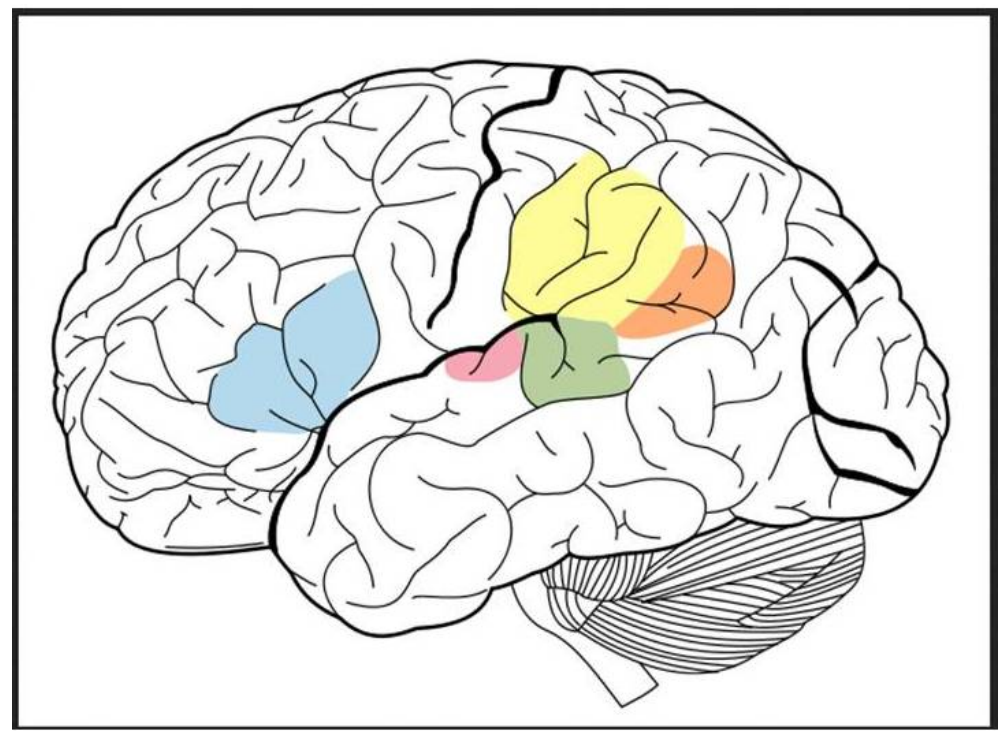

Figure 1. Brain regions important for language. Broca's area (blue), auditory cortex (pink), Wernicke's area (green), Supramarginal gyrus (yellow), angular gyus (orange). (Figure from the wikipedia website http://en.wikipedia.org/ wiki/File:Brain_Surface_Gyri.SVG).

The study of human brain connectivity generally falls under one or more of three categories: structural, functional, and effective [20].

\subsection{DTI structural connectivity studies of brain}

Structural connectivity denotes networks of anatomical (e.g., axonal) links) for which the primary goal is to understand what brain structures are capable of influencing each other via direct or indirect axonal connections. Structural connectivity might be studied in vivo using invasive axonal labeling techniques or noninvasive MRI-based diffusion weighted imaging (DWI/DTI) methods. These methods cannot measure individual axons but can measure the water diffusion signal from a group of axons that have parallel geometric properties within a fiber bundle. DTI connectivity is influenced by the number of axons and the amount of myelination within the fiber bundle.

Diffusion Tensor Imaging (DTI) tractography is a neuroimaging technique that allows for the virtual dissection of fiber tracts in the living brain based on the directionally biased diffusion of water in white matter [21]. DTI analysis provides several parameters that quantify the properties of the fiber bundle: fractional anisotropy ( a measure of the amount of anisotropy of water diffusion between the primary fiber direction and the perpendicular to the primary fiber direction); axial water diffusion diffusivity ( the amount of water diffusion along the primary direction of the fiber bundle); radial diffusivity ( the amount the water diffusion perpendicular to the primary direction of the fiber bundle); mean diffusivity (characterizes the 
overall mean-squared displacement of water molecules); relative anisotropy; and volume ratio. These parameters can be calculated on a voxel by voxel basis within the DTI image The exact equations used to calculate these DTI parameters have been published by LeBihan et al [22]. Other important parameters that characterize the fiber bundle are the tractography analysis which is a procedure to demonstrate the neural tracts[23]. These neural tracts have properties such as mean fiber length, fiber volume, and mean FA within the fiber tract. This tractography analysis can be used to measure connectivity between specific regions of the brain such as between Broca's area and Wernicke's area or other language-related brain regions. The figures that follow (Figures $2 \mathrm{~A}, 2 \mathrm{~B}$, and 2C) show an example of fibers tract4s connected to Broca's area in the left hemisphere.

DTI [24-27] has been used to study language connections. For example, DTI studies have identified association between variation in white matter microstructure and differences in reading skill [28] [29] [30]. Klingberg et al [30] found that white matter diffusion anisotropy in the temporo-parietal region of the left hemisphere was significantly correlated with reading scores within the reading-impaired adults and within the control group. Nioqi et al [28] found strong correlation between fractional anisotropy (FA) values in a left temporo-parietal white matter region and standardized reading scores of typically developing children. Deutsch et al [29] found that white matter structure (as measured by fractional anisotropy) and coherence index (CI) significantly correlated with behavioral measurements of reading, spelling, and rapid naming performance in children. Glasser et al used Diffusion Tensor Imaging (DTI) tractography to detect leftward asymmetries in the arcuate fasciculus [31]. The arcuate fasciclus is a pathway that links temporal and inferior frontal language cortices and is divided into 2 segments with different hypothesized functions, one terminating in the posterior superior temporal gyrus (STG) and another terminating in the middle temporal gyrus (MTG). STG terminations were strongly left lateralized and overlapped with phonological activations in the left but not the right hemisphere, suggesting that only the left hemisphere phonological cortex is directly connected with the frontal lobe via the arcuate fasciculus. MTG terminations were also strongly left lateralized, overlapping with left lateralized lexical--semantic activations. Smaller right hemisphere MTG terminations overlapped with right lateralized prosodic activations. They used a recent model of brain language processing to explain 6 aphasia syndromes [31].These studies demonstrate the potential for using DTI to measure white matter structural changes in dyslexia.

\subsection{Brain studies of functional and effective connectivity}

Functional connectivity denotes symmetrical correlations in activity between brain regions during information processing. Here the primary goal is to understand which regions are functionally related through correlations in their activity, as measured by some imaging technique. Functional connectivity is a powerful noninvasive technique used to investigate the distribution of neural networks in healthy participants and affected subjects, which can be characterized by low-frequency fluctuations in the BOLD signal when the subject is performing a task $[32,33]$. The BOLD response of a continuous task leads to coherent signal changes in anatomically different, but functionally connected, brain structures and thus implies the 


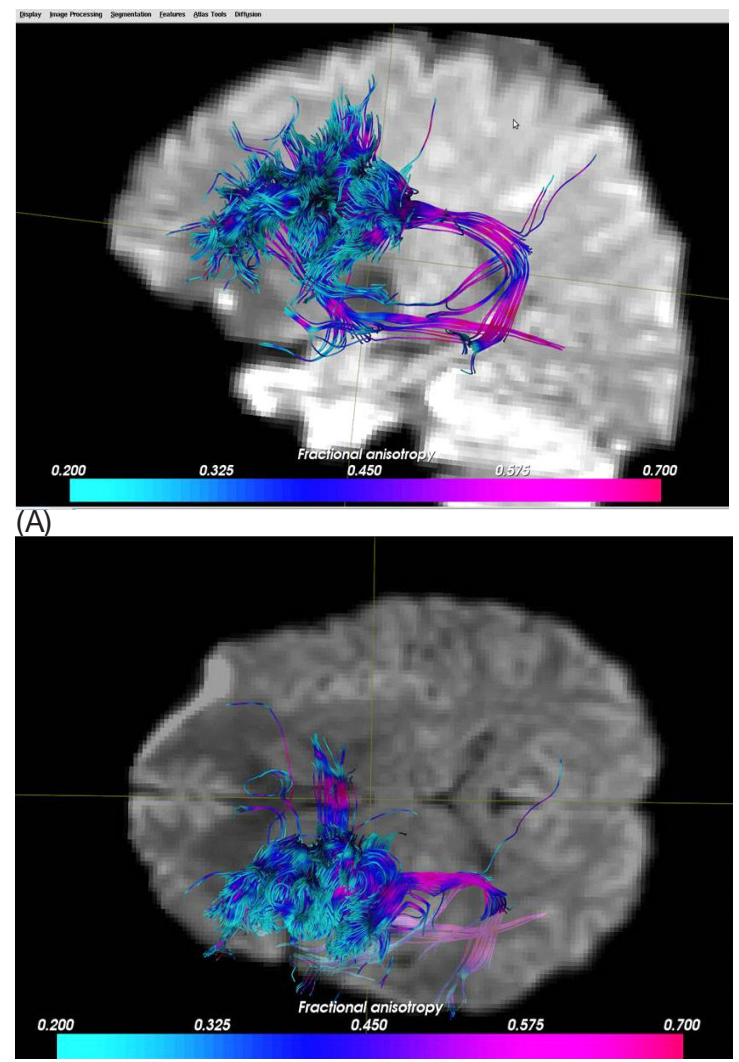

(B)

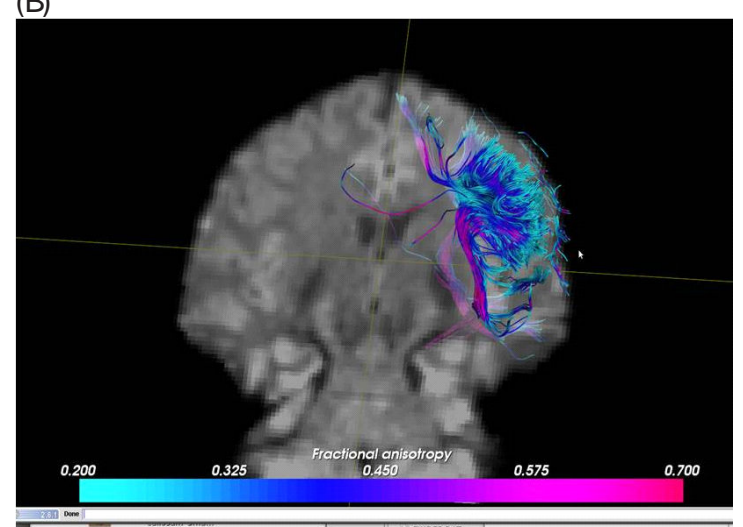

(C)

Figure 2. DTI fiber tracts connected to Broca's area. Sagittal view (part A), axial view (part B), and coronal view (part C) showing fibers in the frontal and temporal lobe. The color coding of the fibers is related to the amplitude of the fractional anisotropy within the fiber. A color scale bar is shown at the bottom. 
existence of neuronal connections between these regions. Coherent signal changes in anatomically different brain structures imply the existence of neuronal connections between these regions. Exploratory data analysis methods have the attractive feature of being model free and thus allowing unbiased studies of brain signal responses.

Examples in fMRI/PET include principal component analysis (PCA), independent component analysis (ICA), and cluster analysis. There are also model-free analyses of interregional connectivity [34-41]. A popular form of functional connectivity analysis using functional magnetic resonance imaging (fMRI) has been to compute the pairwise correlation (or partial correlation) in BOLD activity for a large number of voxels or regions of interest within the brain volume. The figure 3 below shows an example pair of BOLD signals that have a high degree of correlation. For example functional MRI connectivity can be used to study the functional signal correlations between Broca's area and Wernicke's area.

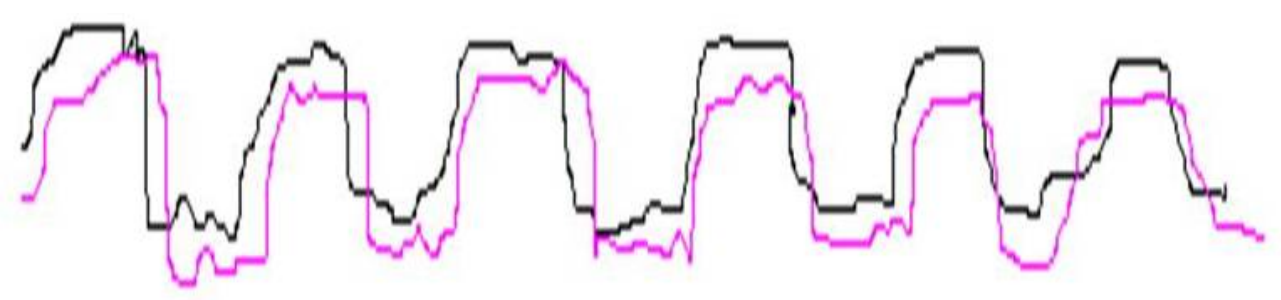

Figure 3. Example of the time course of fMRI signals from two different brain regions which are functionally connected. Notice that the two signals (black and red lines) are closely correlated but not exactly the same.

In contrast to the symmetric nature of functional connectivity, effective connectivity denotes asymmetric or causal dependencies between brain regions. Here the primary goal is to identify which brain structures in a functional network are causally influencing other elements of the network during some stage or form of information processing. Often the term "information flow" is used to indicate directionally specific (although not necessarily causal) effective connectivity between neuronal structures. Popular effective connectivity methods, applied to fMRI and/or electrophysiological (EEG, iEEG, MEG) imaging data, include dynamic causal modeling, structural equation modeling, transfer entropy, and Granger-causal methods. An example of fMRI connectivity using Broca's area as a seed region is shown below in Figure 4.

\section{Connectivity imaging studies of specific learning disabilities}

\subsection{Functional connectivity studies}

Currently, imaging research studies of dyslexia are moving away from simply localizing taskrelated activation to regions of interest (ROI) to analyzing functional connectivity among different brain regions in specific task environments [42] or resting states [43]. Previous functional connectivity studies of dyslexia were mostly focused on the angular gyrus. Asyn- 


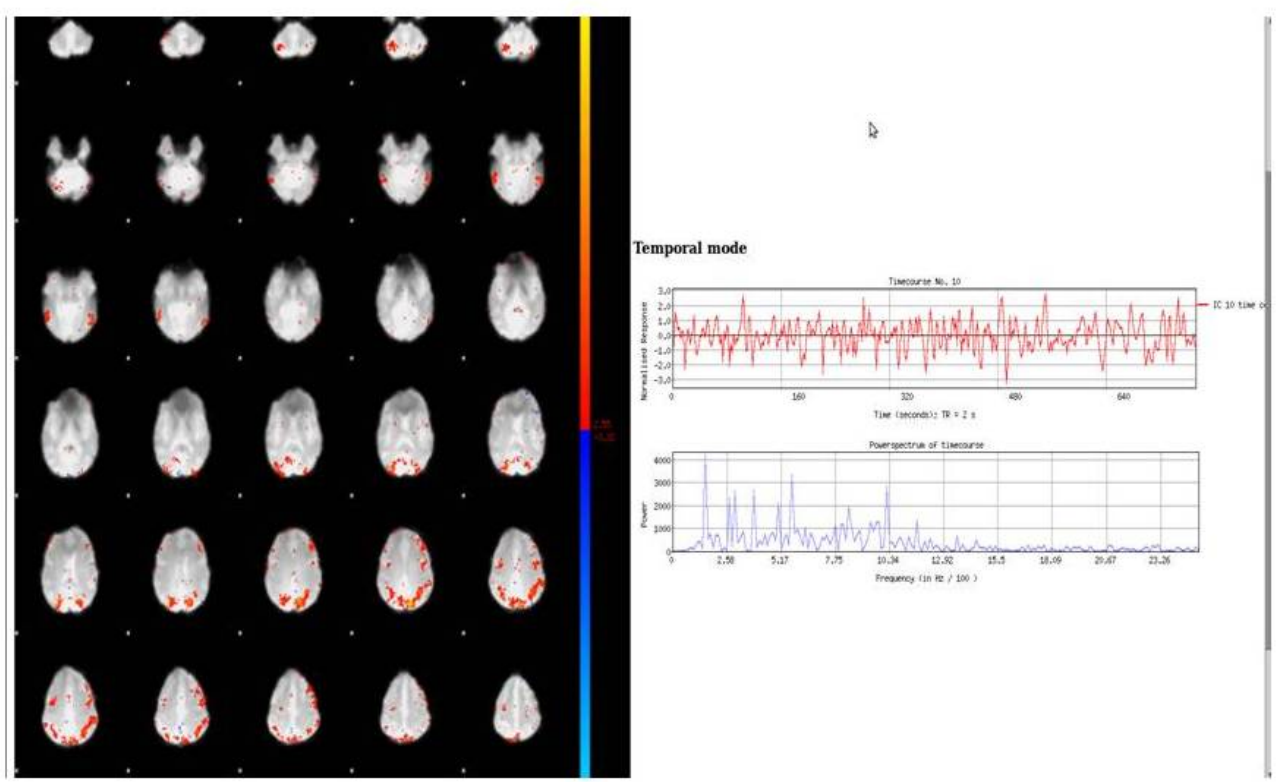

Figure 4. FMRI connectivity analysis related to left-sided Broca's area using FSL's Independent Component Analysis software Melodic combined with UW software. The red plot shows the time course of this ICA component and the plot in blue shows the frequency spectrum. Notice that there are several anatomical regions of the brain that are involved in this component including the left frontal lobe (which includes Broca's area), left and right parietal lobe, left and right temporal lobe.

chrony of regional cerebral blood flow changes in the angular gyrus and extrastriate occipital/ temporal lobe regions suggested functional disconnection during single word reading [44]. Pugh et al [45] showed functional disconnections between the angular gyrus and temporal and occipital areas (namely, lateral extrastriate, medial extrastriate, and primary visual cortex) in the left hemisphere specific to the phonological processing. Shaywitz et al. [46] found functional connections between the occipitotemporal region and inferior frontal gyrus in the left hemisphere in normal readers under a real-word reading condition. Poor readers, in contrast, exhibited more functional connections between the left occipitotemporal region and right middle and inferior frontal gyri [46].

Shaywitz et al documented that the important difference between compensated young adults with a history of dyslexia and young adults who are good readers without a history of dyslexia lies in connectivity among regions rather than in regions of activation per se [46]. Milne et al. [47] reported that an individual with developmental dyslexia showed increased activation, as the phonological processing demands increased, in the left inferior frontal gyrus, right parietal cortex, right occipital cortex, and cerebellum. Both the Shaywitz et al. [46] and Milne et al. [47] studies had shown the importance of connectivity between posterior and anterior language systems in supporting the reading process. Betan et al, [48] have recently used fMRI connectivity to examine task-specific modulations of effective connectivity within a left-hemisphere 


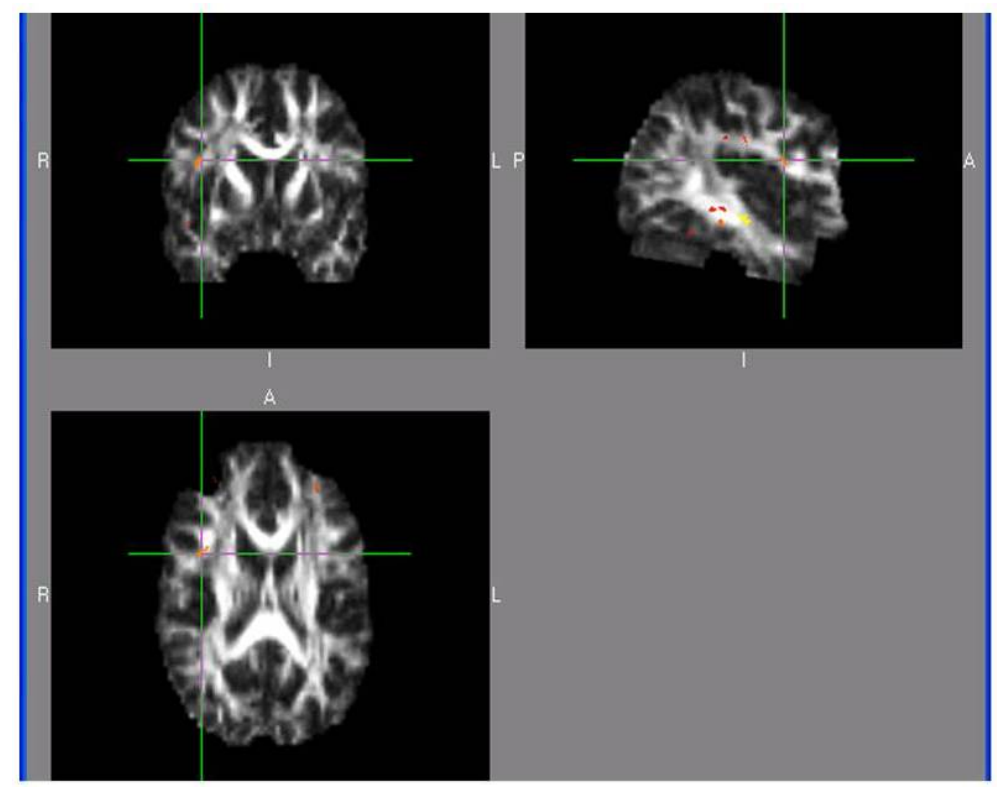

Figure 5. Group differences for controls > dyslexics in analysis of fractional anisotropy with FSL-based TBSS software. Crosshair on a significant cluster near $\mathrm{R}$ inf. frontal gyrus.

language network during spelling and rhyming judgments on visually presented words. They used dynamic causal modeling to show that each task preferentially strengthened modulatory influences converging on its task-specific site (LTC for rhyming, IPS for spelling). Their findings also showed that switching tasks led to changes in the target area influenced by the IFG, suggesting that the IFG may play a pivotal role in setting the cognitive context for each task [48].

\subsection{Converging fMRI and DTI Imaging findings}

Our first DTI Study [49] identified differences between adults with and without dyslexia (which is also a writing disorder, [50]) in the right inferior gyrus (See Figure 5). This is one of the same regions where structural differences were found between dyslexics and good readers in an MRI structural study (Eckert et al., 2003) [51] and the same region where functional differences were found in an fMRI orthographic task contrast before but not after orthographic treatment (Richards et al., 2006a) [52]. Trends towards less activation in right inferior frontal gyrus were associated with improved phonological decoding following treatment (Richards et al., 2006b) [53].

Trends towards less activation in right inferior frontal gyrus were associated with improved phonological decoding following treatment [53]. These findings suggest that right inferior frontal gyrus plays a role in orthographic coding, a process which our behavioral studies for nearly two decades have shown contributes uniquely to handwriting, spelling, and composi- 


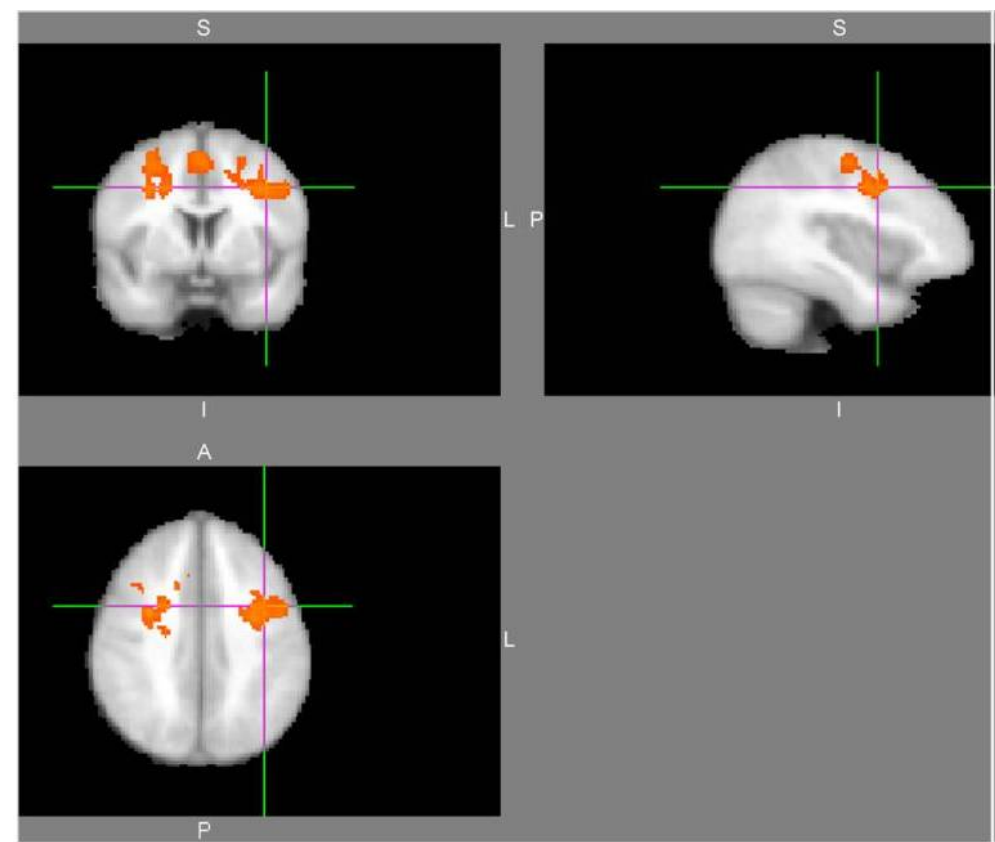

Figure 6. Group difference map for dyslexics greater than controls. The individual maps used in this analysis were correlation maps created when the seed $\mathrm{ROI}$ in the left inferior frontal gyrus was compared to the rest of the brain voxels.

tion[54]. Thus, we predict that in studies in progress children with handwriting disabilities will differ from good writers in the right inferior frontal gyrus.

Differences in functional connectivity were also found between children with and without dyslexia before but not after treatment on a phonological spelling task (phoneme mappingdeciding whether letter(s) in pair of pronounceable nonwords could stand for the same sound[55]. These data were analyzed with a seed point correlational method for functional connectivity from four seed points based on prior studies: inferior frontal gyrus, middle frontal gyrus, the occipital region, and cerebellum. Before treatment, there was a significant difference in fMRI connectivity between children with dyslexia and normal reading controls in the degree of connectivity between left inferior frontal gyrus and the following regions: right and left middle frontal gyrus, right and left supplemental motor area, left precentral gyrus, and right superior frontal gyrus. There were no significant differences when seed regions were placed in the middle frontal gyrus, occipital gyrus or cerebellum. Children with dyslexia had greater functional connectivity from the left inferior frontal gyrus seed point to the right inferior frontal gyrus than did the children without dyslexia as shown in Figure 6.

The children with dyslexia then participated in a 3-week instructional program that provided explicit instruction in linguistic awareness, alphabetic principle (taught in a way to maximize temporal contiguity of grapheme-phoneme associations and to train both phonological and orthographic loops), decoding and spelling. At Time2, the treated children with did not differ 
from the children without dyslexia in any of the clusters in the group. The main result was that children with dyslexia had greater functional connectivity from the left inferior frontal gyrus seed point to the right inferior frontal gyrus than did the children without dyslexia before but not after treatment [55]. Thus, the structural and functional connectivity studies provided converging evidence for abnormalities related to inferior frontal gyrus (on right or left) in children with dyslexia.

\subsection{Stanberry model of fMRI connectivity in dyslexia}

Stanberry et al [35] developed a model of fMRI connectivity based on earlier results that predicts that for normal readers there will be functional connectivity among 5 major readingrelated brain regions: (a) frontal lobe (including the inferior frontal gyrus and middle frontal gyrus); (b) parietal lobe (including the angular gyrus); (c) visual processing areas (including occipitotemporal region); (d) fusiform/lingual word form region; and (e) the cerebellum. This model is generally consistent with that reported by other research groups for normal reading [46]; it is also consistent with phonological loop in verbal working memory as a deficit in dyslexia $[56,57]$. We predicted that individual dyslexics may have impaired connectivity in any one or a combination of these major circuits. In our first fMRI connectivity study, we investigated differences in cortical networks used by adult controls compared to adult dyslexics during the previously described Phoneme Mapping. By definition, functional connectivity refers to a correlation or synchronization between the time courses of activation of two brain regions. We hypothesized that two brain regions that work together have similar temporal response profiles [58]. A model-independent method was used to analyze the timesynchronized activations induced by the phoneme mapping paradigm (adapted from [59]) presented during a continuous task presentation. A standard fMRI acquisition and analysis of the on-off block design was also performed using Phoneme Mapping. Native English speakers ranging in age from 30 to 45 years participated in the connectivity study: 10 healthy righthanded control males (fathers from the family genetics study who did not meet research criteria for dyslexia on tests and also did not have a history of reading problems) and 13 righthanded, otherwise healthy, adult males who did meet the research criteria for dyslexia and had a history of reading and writing problems. The two groups did not differ significantly in mean Verbal IQ [dyslexics, $\mathrm{M}=113.8$ (SD = 10.3); controls, $\mathrm{M}=107.7$ (SD=11.1), but the dyslexics were significantly lower than the control fathers on each of the reading, spelling, and RAN measures.

Structural and functional MR images were collected in accordance with institutional regulations (IRB approval) on a commercial 1.5T MR scanner (General Electric, Waukesha, WI) equipped with echo-speed gradients and a standard birdcage head coil. Functional images were acquired using an echo-planar sequence with imaging parameters set as follows:

"On-Off" task: 20 axial slices, FOV 24cm x 24cm, BW +/- $62.5 \mathrm{kHz}$, TR 2000ms, TE 40ms, Flip $82 \mathrm{deg}$, slice thickness $6 \mathrm{~mm}$, gap $1 \mathrm{~mm}$, resolution $64 \times 64,162$ time points; Continuous task: 20 axial slices, FOV $24 \mathrm{~cm} \times 24 \mathrm{~cm}$, BW +/- $62.5 \mathrm{kHz}$, TR 2000ms, TE $40 \mathrm{~ms}$, Flip $82 \mathrm{deg}$, slice thickness $6 \mathrm{~mm}$, gap $1 \mathrm{~mm}$, resolution $64 \times 64,483$ time points. 
Cardiac and respiratory rates were digitally recorded with a pulse oximeter and a flexible belt, respectively, using a sampling frequency of $100 \mathrm{~Hz}$. Three different seed regions were used for connectivity analysis - right and left inferior frontal gyrus and cerebellum.

For the standard block fMRI acquisition and analysis of controls, fMRI brain activation was detected in the following brain regions: for the right side - inferior frontal gyrus, middle frontal gyrus, cerebellum crus I, cerebellum crus II, occipital gyrus, superior parietal gyrus, inferior parietal gyrus, angular gyrus, lingual gyrus, and fusiform gyrus; for the left side - superior parietal gyrus, angular, occipital gyrus, cerebellum crus I, cerebellum crus II, lingual.

For the fMRI connectivity analysis of the continuous phoneme mapping paradigm, we narrowed the five region model above to a focus on three regions based on structural MRI differences in dyslexics from a family genetics study [51]. Results showed that (a) when the right IFG was chosen as the seed region, significant differences $(p<.05)$ were found between dyslexics and controls in right inferior frontal triangularis, bilateral fusiform, bilateral middle and inferior occipital gyri, right angular gyrus, bilateral ITG and cerebellum; (b) when the left IFG was chosen as the seed region, significant differences $(p<.05)$ were found between dyslexics and controls in the following brain regions: right inferior frontal triangularis, right middle occipital gyrus, right inferior occipital gyrus, and right cerebellum (VI); and (c) when the cerebellum was chosen as the seed region, significant differences $(p<.05)$ were found between dyslexics and controls in the following brain regions: bilateral superior frontal gyrus, left middle frontal gyrus, right angular gyrus, and right middle occipital gyrus. Adult dyslexics, when compared to controls, had impaired cortical connections in brain regions important for phonological processing. The abnormality in functional connectivity from cerebellum in dyslexics may be related to Klingberg et al.'s [30] finding, based on DTI, that white matter diffusion anisotropy in the temporo-parietal region of the left hemisphere is significantly correlated with reading in normal and dyslexic readers. Insufficient myelination of the axonal pathways is one possible explanation for the low anisotropy index values observed in poor readers [60]. Structural abnormalities in white matter pathways could interfere with neuronal transmission, which will directly affect the synchrony of the BOLD signal. Of most importance, functional disconnections were also observed when seed regions were set in bilateral IFG. Bilateral IFG and right cerebellum were found to be abnormal in child dyslexics compared to normal controls ascertained using the same research criteria in our structural MRI studies [51]. Also see Berninger, Raskind, Richards et al. [50].

\section{Future perspectives}

One of the great potential techniques in this area of language connectivity analysis is the integration of both functional and structural connectivity as shown by Morgan et al [61]. They measured connections between Wernicke's (WA), Broca's (BA) and supplementary motor area (SMA). Along the path between BA and SMA, they showed that fibers tracked measured from DTI generally formed a single bundle and the mean radius of the bundle was positively correlated with functional connectivity. They concluded that the insights gained from this 
work offers a useful guidance for non-invasive means to evaluate brain network integrity in vivo for use in diagnosing and determining disease progression and recovery [61]. The concept of integrating information across brain imaging modalities will allow the study of human language network as a systems approach. Another futuristic concept has been described by Rota et al [62] where they discuss the mechanisms of cortical reorganization underlying the enhancement of speech. They were able to measure changes in functional and effective connectivity induced in subjects who learned to deliberately increase activation in the right inferior frontal gyrus [62]. Also, see [63] for a model of the four multi-leveled functional language systems, which provides the conceptual framework for testing a model that differentiates among typical oral and written language learners (OWLs), dysgraphia, dyslexia, and OWL LD at the behavioral (phenotype and response to instruction) and brain levels of analysis.

\section{Conclusions}

The language connectivity findings discussed in this chapter suggest that structural and functional connectivity are adding and will continue to add to our understanding of language and language learning. There are specific language pathways and connections that are crucial for language acquisition and function. The integrity of these connections can be tested using structural DTI and functional MRI connectivity imaging. Individuals with learning and language disabilities have been reported to have different fMRI and DTI measurable connections than those with normal language functions. Once the techniques have been fully tested and developed, the application of language connectivity techniques to the individual assessment, treatment design, and response to treatment would also have enormous practical applications in the clinic and schools.

\section{Acknowledgements}

This project received support from the NIH/NICHD Grant 1P50HD071764 (overall PI Virginia Berninger, PI of project 3 Todd Richards).

\section{Author details}

Todd L. Richards ${ }^{1 *}$ and Virginia W. Berninger ${ }^{2}$

*Address all correspondence to: toddr@u.washington.edu

1 Department of Radiology, University of Washington Medical Center, Seattle, WA, USA

2 Department of Educational Psychology, University of Washington, Seattle, WA, USA 


\section{References}

[1] Liberman A. The reading researcher and the reading teacher need the right theory of speech. Scientific Studies of Reading 1999;3:95-111.

[2] Berninger V, Graham S. Language by hand: A synthesis of a decade of research on handwriting. Handwriting Review 1998;12:11-25.

[3] Berninger V. Development of language by hand and its connections to language by ear, mouth, and eye. Topics in Language Disorders, 20, 65-84 2000;20:65-84.

[4] Trask RL. Language: The Basics. New York: Routledge; 1999.

[5] Lesser R. Language in the Brain: Neurolinguistics, An Encyclopedia of Language. New York: Routledge; 1989.

[6] Binder JR, Frost JA, Hammeke TA, Cox RW, Rao SM, Prieto T. Human brain language areas identified by functional magnetic resonance imaging. J Neurosci 1997;17:353-362.

[7] Binder JR, Frost JA, Hammeke TA, Bellgowan PS, Springer JA, Kaufman JN, et al. Human temporal lobe activation by speech and nonspeech sounds. Cereb Cortex 2000;10(5):512-28.

[8] Cannestra AF, Bookheimer SY, Pouratian N, O'Farrell A, Sicotte N, Martin NA, et al. Temporal and topographical characterization of language cortices using intraoperative optical intrinsic signals. Neuroimage 2000;12(1):41-54.

[9] Castillo EM, Simos PG, Davis RN, Breier J, Fitzgerald ME, Papanicolaou AC. Levels of word processing and incidental memory: dissociable mechanisms in the temporal lobe. Neuroreport 2001;12(16):3561-6.

[10] Jancke L, Wustenberg T, Scheich H, Heinze HJ. Phonetic perception and the temporal cortex. Neuroimage 2002;15(4):733-46.

[11] McDermott KB, Petersen SE, Watson JM, Ojemann JG. A procedure for identifying regions preferentially activated by attention to semantic and phonological relations using functional magnetic resonance imaging. Neuropsychologia 2003;41(3):293-303.

[12] Bookheimer S. Functional MRI of language: new approaches to understanding the cortical organization of semantic processing. Annu Rev Neurosci 2002;25:151-88.

[13] Hickok G, Poeppel D. Dorsal and ventral streams: a framework for understanding aspects of the functional anatomy of language. Cognition 2004;92(1-2):67-99.

[14] Price CJ, Mummery CJ, Moore CJ, Frakowiak RS, Friston KJ. Delineating necessary and sufficient neural systems with functional imaging studies of neuropsychological patients. J Cogn Neurosci 1999;11(4):371-82. 
[15] Paulesu E, Goldacre B, Scifo P, Cappa SF, Gilardi MC, Castiglioni I, et al. Functional heterogeneity of left inferior frontal cortex as revealed by fMRI. Neuroreport 1997;8(8):2011-7.

[16] Broca P. Nouvelle observation d'aphemie produite par une lesion de la troisieme circonvolution frontale. Bulletins de la Societe anatomie (Paris), 2e serie 1861;6:398-407.

[17] Wernicke C. The symptom complex of aphasia (1874). Reprinted in English in Proc. Boston Colloq. Philos. Sci. 1874;4:34-97.

[18] Dejerine J. Anatomy of central nervous system. Paris: Masson; 1895.

[19] Mullen T. SIFT Online Handbook and User Manual. http://sccn.ucsd.edu/wiki/SIFT 2010.

[20] Bullmore E, Sporns O. Complex brain networks: graph theoretical analysis of structural and functional systems. Nat Rev Neurosci 2009;10(3):186-98.

[21] Beaulieu C. The basis of anisotropic water diffusion in the nervous system - a technical review. NMR Biomed 2002;15(7-8):435-55.

[22] Le Bihan D, Mangin JF, Poupon C, Clark CA, Pappata S, Molko N, et al. Diffusion tensor imaging: concepts and applications. Journal of magnetic resonance imaging: JMRI 2001;13(4):534-546.

[23] Filler AI. The History, Development and Impact of Computed Imaging in Neurological Diagnosis and Neurosurgery: CT, MRI, and DTI. Internet Journal of Neurosurgery 2010;7(1).

[24] Catani M, Mesulam M. The arcuate fasciculus and the disconnection theme in language and aphasia: history and current state. Cortex 2008;44(8):953-61.

[25] Nucifora PG, Verma R, Melhem ER, Gur RE, Gur RC. Leftward asymmetry in relative fiber density of the arcuate fasciculus. Neuroreport 2005;16(8):791-4.

[26] Parker GJ, Luzzi S, Alexander DC, Wheeler-Kingshott CA, Ciccarelli O, Lambon Ralph MA. Lateralization of ventral and dorsal auditory-language pathways in the human brain. Neuroimage 2005;24(3):656-66.

[27] Powell HW, Parker GJ, Alexander DC, Symms MR, Boulby PA, Wheeler-Kingshott $\mathrm{CA}$, et al. Hemispheric asymmetries in language-related pathways: a combined functional MRI and tractography study. Neuroimage 2006;32(1):388-99.

[28] Niogi SN, McCandliss BD. Left lateralized white matter microstructure accounts for individual differences in reading ability and disability. Neuropsychologia 2006;44: 2178-2188.

[29] Deutsch GK, Dougherty RF, Bammer R, Siok WT, Gabrieli JD, Wandell B. Children's reading performance is correlated with white matter structure measured by diffusion tensor imaging. Cortex 2005;41:354-363. 
[30] Klingberg T, Hedehus M, Temple E, Salz T, Gabrieli JD, Moseley ME, et al. Microstructure of temporo-parietal white matter as a basis for reading ability: evidence from diffusion tensor magnetic resonance imaging [see comments]. Neuron 2000;25(2):493-500.

[31] Glasser MF, Rilling JK. DTI tractography of the human brain's language pathways. Cereb Cortex 2008;18(11):2471-82.

[32] Hampson M, Peterson B, Skudlarski P., Gatenby J, Gore J. Detection of functional connectivity using temporal correlations in MR images. Human Brain Mapping 2002;15:247-262.

[33] Lowe M, Mock B, Sorenson J. Functional connectivity in single and multislice echoplanar imaging using resting-state fluctuations. Neuroimage 1998;7:119-132.

[34] Stanberry L, Nandy R, Cordes D. Cluster analysis of fMRI Data using Dendrogram Sharpening. Human Brain Mapping 2003;20:201-219.

[35] Stanberry LI, Richards T, Berninger VW, Stock P, Nandy RR, Aylward E, et al. Low Frequency Signal Changes Reflect Differences in Functional Connectivity between Good Readers and Dyslexics during Continuous Phoneme Mapping. Magnetic Resonance Imaging 2006;24:217-229.

[36] Nandy R, Cordes D. Improving the spatial specificity of canonical correlation analysis in fMRI. Magn Reson Med 2004;52(4):947-52.

[37] Nandy RR, Cordes D. Novel nonparametric approach to canonical correlation analysis with applications to low CNR functional MRI data. Magn Reson Med 2003;50(2): 354-65.

[38] Nandy RR, Cordes D. Novel ROC-type method for testing the efficiency of multivariate statistical methods in fMRI. Magn Reson Med 2003;49(6):1152-62.

[39] Cordes D, Haughton V, Carew JD, Arfanakis K, Maravilla K. Hierarchical clustering to measure connectivity in fMRI resting-state data. Magn Reson Imaging 2002;20(4): 305-17.

[40] Cordes D, Haughton VM, Arfanakis K, Carew JD, Turski PA, Moritz CH, et al. Frequencies contributing to functional connectivity in the cerebral cortex in "restingstate" data. AJNR Am J Neuroradiol 2001;22(7):1326-33.

[41] Cordes D, Haughton VM, Arfanakis K, Wendt GJ, Turski PA, Moritz CH, et al. Mapping functionally related regions of brain with functional connectivity MR imaging [In Process Citation]. AJNR Am J Neuroradiol 2000;21(9):1636-44.

[42] Büchel C, Coull, J., Friston, K. The predictive value of changes in effective connectivity for human learning. Science 1999;283:1538-1540. 
[43] Cordes D, Haughton VM, Arfanakis K, Wendt GJ, Turski PA, Moritz CH, et al. Mapping functionally related regions of brain with functional connectivity MR imaging. AJNR Am J Neuroradiol 2000;21(9):1636-44.

[44] Horwitz B, Rumsey JM, Donohue BC. Functional connectivity of the angular gyrus in normal reading and dyslexia. Proc Natl Acad Sci U S A 1998;95(15):8939-44.

[45] Pugh K, Mencl, W., Shaywitz, B., Shaywitz, S., Fulbright, R., Constable, R., Skudlarski, P., Marchione, K., Jenner, A., Fletcher, J., Liberman, A., Shankweiler, D., Katz, L., Lacadie, C., Gore,J. The angular gyrus in developmental dyslexia: Task-specific differences in functional connectivity within posterior cortex. Psychological Science 2000;11:51-56.

[46] Shaywitz SE, Shaywitz BA, Fulbright RK, Skudlarski P, Mencl WE, Constable RT, et al. Neural systems for compensation and persistence: young adult outcome of childhood reading disability. Biol Psychiatry 2003;54(1):25-33.

[47] Milne D, Syngeniotis A, Jackson G, Corballis M. Mixed lateralization of phonological assembly in developmental dyslexia. Neurocase 2002;8:205-209.

[48] Bitan T, Booth JR, Choy J, Burman DD, Gitelman DR, Mesulam MM. Shifts of effective connectivity within a language network during rhyming and spelling. J Neurosci 2005;25(22):5397-403.

[49] Richards T, Stevenson J, Crouch J, Johnson LC, Maravilla K, Stock P, et al. Tractbased spatial statistics of diffusion tensor imaging in adults with dyslexia. AJNR Am J Neuroradiol 2008;29(6):1134-9.

[50] Berninger VW, Raskind W, Richards T, Abbott R, Stock P. A multidisciplinary approach to understanding developmental dyslexia within working-memory architecture: genotypes, phenotypes, brain, and instruction. Dev Neuropsychol 2008;33(6): 707-44.

[51] Eckert MA, Leonard CM, Richards TL, Aylward EH, Thomson J, Berninger VW. Anatomical correlates of dyslexia: frontal and cerebellar findings. Brain 2003;126(Pt 2): 482-494.

[52] Richards T, Aylward E, Berninger V, Field K, Parsons A, Richards A, et al. Individual fMRI activation in orthographic mapping and morpheme mapping after orthographic or morphological spelling treatment in child dyslexics. Journal of Neurolinguistics 2006;19:56-86.

[53] Richards T, Aylward E, Raskind W, Abbott R, Field K, Parsons A, et al. Converging evidence for triple word form theory in child dyslexia. Special Issue on Brain Imaging in Developmental Neuropsychology 2006;in press.

[54] Berninger V, Richards T. Inter-relationships among behavioral markers, genes, brain and treatment in dyslexia and dysgraphia. Future Neurol 2011;5(4):597-617. 
[55] Richards TL, Berninger VW. Abnormal fMRI Connectivity in Children with Dyslexia During a Phoneme Task: Before But Not After Treatment. J Neurolinguistics 2008;21(4):294-304.

[56] Heilman KM, Voeller K, Alexander AW. Developmental dyslexia: a motor-articulatory feedback hypothesis. Ann Neurol 1996;39(3):407-12.

[57] Chen SH, Desmond JE. Cerebrocerebellar networks during articulatory rehearsal and verbal working memory tasks. Neuroimage 2005;24:332-338.

[58] Koshino H, Carpenter PA, Minshew NJ, Cherkassky VL, Keller TA, Just MA. Functional connectivity in an fMRI working memory task in high-functioning autism. Neuroimage 2005;24(3):810-21.

[59] Aylward EH, Richards TL, Berninger VW, Nagy WE, Field KM, Grimme AC, et al. Instructional treatment associated with changes in brain activation in children with dyslexia. Neurology 2003;61(2):212-9.

[60] Wimberger DM, Roberts TP, Barkovich AJ, Prayer LM, Moseley ME, Kucharczyk J. Identification of 'premyelination" by diffusion-weighted MRI. J Comput Assist Tomogr 1995;19:28-33.

[61] Morgan VL, Mishra A, Newton AT, Gore JC, Ding Z. Integrating functional and diffusion magnetic resonance imaging for analysis of structure-function relationship in the human language network. PLoS One 2009;4(8):e6660.

[62] Rota G, Handjaras G, Sitaram R, Birbaumer N, Dogil G. Reorganization of functional and effective connectivity during real-time fMRI-BCI modulation of prosody processing. Brain Lang 2011;117(3):123-32.

[63] Berninger V, Niedo J. Individualizing instruction for students with oral and written language difficulties. In: Mascolo J, Flanagan D, Alfonso V, editors. Essentials of planning, selecting and tailoring intervention: Addressing the needs of unique learners. New York: Wiley; 2013. In Press 
